\title{
Contraceptives and HIV transmission
}

To the editor - A recently reported study in macaques raised the possibility that use of contraceptives containing only progestins, such as Depo-Provera, may increase the likelihood of HIV transmission to women ${ }^{1}$. On considering a broader literature on the subject, we believe that it is difficult to arrive at any conclusion concerning the impact of such contraceptives on HIV transmission.

In the Marx et al. study, 14 out of 18 macaques pretreated with progesteronereleasing pellets and then inoculated vaginally with SIV became infected compared to one of ten macaques pre-treated with placebo pellets and inoculated during the follicular phase of the menstrual cycle; a 7.8-fold increase in infection. In separate experiments (described in the Methods section of the paper) employing the same protocol, viral stocks and doses, cycling macaques were exposed to SIV without regard to the phase of their menstrual cycles. Four of 11 animals were infected. The infection rate seen in the progesterone-pretreated macaques was 2.1 fold $(95 \%$ C.I. $0.9-4.9)$ higher than that of these cycling macaques. This lower estimate of the risk associated with exogenous progesterone used viral exposures timed to more closely parallel those of women, who are likely to be exposed to HIV throughout their menstrual cycle including the luteal phase when the vaginal epithelium is naturally thinner than during the follicular phase.

In our preliminary analysis of women known to be exposed to an HIV-infected sexual partner, the infectivity (probability of HIV transmission per act of intercourse) for Depo-Provera use was similar to that for use of neither hormonal nor barrier contraceptive measures ${ }^{2}$. Ten published human studies have examined the relationship between Depo-Provera use and HIV transmission (references available). These studies gave estimates of risk (odds ratio or relative risks) ranging from 0.44 to 3.8 for those taking Depo-Provera; seven gave risks between 1.0 and 2.0. Only one risk estimate remained significant after adjusting for confounding factors. The results from cross-sectional studies are difficult to interpret since one does not know whether exposure to HIV occurred and, if it occurred, whether it coincided with the period of Depo-Provera use. Two prospective studies have evaluated the risk of HIV seroconversion among women who were using DMPA at baseline or during followup; their interpretation is limited by the absence of any data on HIV exposure.

Taken together, the macaque study and these epidemiological studies suggest that the effect of Depo-Provera use on HIV transmission will be difficult to establish. In the epidemiological studies, where differences between risk in the DepoProvera-exposed and the unexposed groups is two fold or less, bias is always a potential issue. Biases in studies of contraceptive use and HIV transmission are particularly important to consider since both the choice of contraceptive method and the risk of HIV infection are related to sexual behaviors. For example, women who use hormonal contraceptives may be at increased risk of HIV simply due to reduced use of condoms. Although large and well-defined studies may clarify the relative roles of biology and bias, questions concerning the relationship between Depo-Provera use and HIV transmission will likely remain for years. Certainly there are no data to suggest that Depo-Provera protects against HIV transmission, and therefore, all couples at risk of HIV infection and engaging in sexual intercourse need to use condoms consistently and correctly regardless of any other contraceptive they may use.

Ann Duerr, D. Warren \& D. SMITH Centers for Disease Control and Prevention Atlanta, Georgia 30341-3724, USA

\section{T. NAGACHINTA \\ The CONRAD Program/USAID, \\ Atlanta, Georgia, USA}

Marx replies - We agree with the above opinions regarding the information gleaned from the literature and from their studies on Depo-Provera. David Satcher (Director of the Centers for Disease Control and Prevention) stresses that any woman who wishes to control fertility and has found a suitable contraceptive method should continue it but dual methods should be used for persons who are at risk of HIV infection. Only abstinence and male latex condoms have been shown to effectively prevent sexual transmission of HIV. In fact, the U.S. Food and Drug Administration already requires that nonbarrier contraceptives must include labeling stating that they do not provide protection against HIV infection. Duerr et al. point out that the effects of progestins on HIV transmission will be difficult to determine. This is also true and is the reason we used the monkey model. This model provides the opportunity to test hypotheses in a controlled manner that would be impossible in human subjects. Duerr et al. also indicate that the risk of SIV infection throughout the menstrual cycle may be less than the 7.7 fold we reported. This is a possible interpretation of some of our data, but was not in the design of the study and sufficient animals were not used to study this in particular. Our study only tested a hypothesis that progesterone enhanced vaginal transmission of SIV and that hypothesis was proven. The question posed by Duerr et al. is being addressed in our new study.

\section{PRESTON A. MARX}

New England Regional Primate Research Center Harvard Medical School, Southborough, Massachussetts 01772-9102, USA

1. Marx, P. et al. Progesterone implants enhance SIV transmission and early virus load. Nature Med. 2, 1084-1089 (1996).

2. Nagachinta, T. et al. HIV infectivity by contraceptive method from a partner study in Northern Thailand. 11th Annual International Conference on AIDS, Vancouver, July 1996.

\section{Mitochondrial mutations and male infertility}

To the editor - Johns has correctly associated mitochondrial mutations with a number of debilitating diseases ${ }^{1}$. We are currently investigating whether mitochondrial mutations represent a significant cause of sperm dysfunction and hence male infertility.

There is one documented case of maternally inherited point mutations resulting in encephalomyopathy and reduced sperm motility (asthenozoo- spermia) ${ }^{2}$. However, little other evidence exists. Yet, we believe that sporadic deletions account for the majority of mtDNA deletions ( $\triangle \mathrm{mtDNA}$ ) associated with asthenozoospermia. It is likely that these $\triangle \mathrm{mtDNA}$ arise via reactive oxygen species (ROS) damage, perhaps generated by the mitochondria themselves or through mitochondrial degradation, an early indicator of apoptosis. Indeed, bax 